\title{
A Seiv Vaccination Model with General Non-Linear Incidence Rate and Waning Preventive Vaccine
}

\author{
A.J. Abdulrazak ${ }^{1}$, M.O. Ibrahim ${ }^{2}$, I.O. Usman ${ }^{1}$ \\ ${ }^{1}$ Department of Mathematics, Usmanu Danfodiyo University Sokoto, Sokoto State, Nigeria \\ ${ }^{2}$ Department of Mathematics, University of Ilorin, Kwara State, Nigeria.
}

\begin{abstract}
This study adopted and analyzed a SEIV (Susceptible, Exposed, Infected, and Vaccinated) vaccination model with general non-linear incidence rate and waning preventive vaccine. Thereafter the Basic Reproduction Number of the disease free equilibrium was found and simulated, it was found to be globally asymptotically stable. The epidemic free equilibrium was also found, analyzed using the Bellman and Cooke's Theorem. The result of the analyses was simulated using MATLAB and found that it is Stable on probability points of the vaccine waning period from 1.0 down to 0.3 and unstable on the points 0.2 and 0.1 . Which is logical, that is, as the vaccine efficacy decreases so does the stability of the model.
\end{abstract}

Keywords: Basic Reproductive Number, Disease Free Equilibrium, Epidemic Free Equilibrium, Stability, Vaccine.

\section{Introduction}

One of the primary reasons of studying infectious diseases is to improve control and ultimately eradicate the infection from the population. Models can be powerful tools in this approach, allowing us to optimize the use of limited resources or simply to target control measures more efficiently. Several forms of control form measures exist all operate by reducing the average amount of transmission between infectious and susceptible individuals. Which control strategy (or mixture of strategies) is used will depend on the disease, the host and the scale of the epidemic. The practice of vaccination began with Edward Jenner in 1796 who developed vaccines against smallpox - which remains the only disease to date that has been eradicated worldwide. Vaccination acts by stimulating a host immune response, such that immunized individuals are protected against infection. In 1988, the World Health Organization (WHO) resolved to use similar campaigns to eradicate polio world-wide by 2005- this is still ongoing work although much progress has been made to date. [7]. It may be noted that the outbreak of infectious diseases cause mortality of millions of people as well as expenditure of enormous amount of money in health care and disease control, it is, therefore, essential that adequate attention must be paid to stop the spread of such diseases by taking control measures, vaccination is an important control measure to reduce the spread of such diseases [1].

Various modelling studies have been made to study the role of vaccination on the spread of infectious diseases in a population. Among the uncountable literature on vaccination models are [1-5, 8-11]

Naresh et al. [1] proposed and analyzed a non-linear mathematical for the spread of carrier dependent infectious diseases in a population with variable sized structure including the role of vaccination. Farrington [2] analyzed the impact of vaccination programme on the transmission potential of the infection in large population and derived relation between vaccine efficacy against transmission, vaccine coverage and reproductive number. Gumel and Moghadas [3] proposed a model for the dynamic of an infectious disease in the presence of a preventive vaccine considering non-linear incidence rate and found the optimal vaccination threshold needed for disease control and eradication. Kribs-Zaleta and Valasco-Hanandez [4] presented a simple two dimensional SIS model with vaccination exhibiting backward bifurcation. Shulgin et al. [5] studied a simple SIR epidemic model with pulse vaccination and shows that pulse vaccination leads to epidemic eradication if certain conditions regarding the magnitude of vaccination proportion and on the period of pulse are satisfied. Jianwen and Ping [8] established an SVEIR epidemic model with non-linear incidence rate under the assumption that vaccinated individuals have partial immunity; they obtained the basic reproductive number, they proved the global asymptotical stability of the disease free equilibrium by Ruth Hurwitz criterion and they obtained the sufficient condition for the global asymptotical stability of the endemic equilibrium. De La Sen et al. [9] presented a linear vaccination for a SEIR propagation disease model. They used a state observer to estimate the true various partial populations of Susceptible, Infected, Infectious and Immune which are assumed to be unknown. De La Sen et al. [10] also proposed a disease free and endemic equilibrium points of SVEIRS epidemic models. Arino et. al [11] proposed a model that looks at the interplay of vaccination strategy together with vaccine efficacy and waning, in particular, it shows that a backward bifurcation leading to bistability can occur. Under mild parameter constraints, compound matrices are used to show that each orbit limits to an equilibrium. In case of bistability, the global result requires novel approach since there is no compact absorbing set. Naresh et. al, [1] extended a 
paper presented by Singh et. al, [12] Gosh et-al [13] by incorporating the effect of vaccine on the spread of carrier infectious diseases and assuming a generalized logistic model governing the growth of carrier population. They used a more realistic standard action type interaction for direct contacts between susceptible and infective instead of simple mass action.

\section{The Mathematical Model}

This mathematical model is a review of Li-Ming Cai and Xue-Zhi Li [6] vaccination model. We adopted the model and carried out a different analysis to test for the epidemic free equilibrium of the model. We still considered the four compartmental model which consists of the Susceptible Individuals (S), Exposed individuals infected but not infectious (E), Infected individuals (I) and Vaccinated individuals (V) With general non-linear incidence rate and waning preventive vaccine.

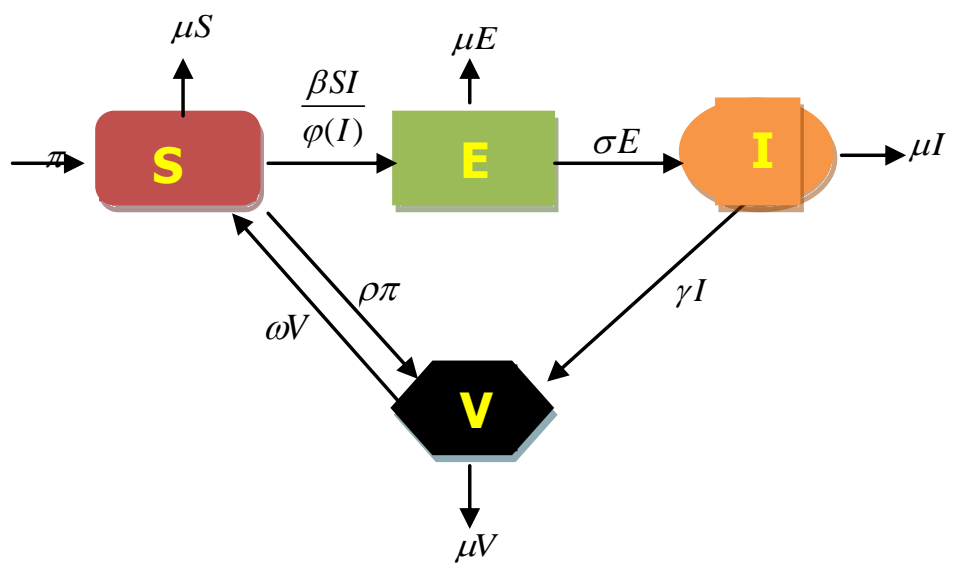

Fig 1. The Model Diagram

$$
\begin{aligned}
& \frac{d S(t)}{d t}=(1-\rho) \pi-\frac{\beta S(t) I(t)}{\varphi(I)}-\mu S(t)+\omega V(t) \\
& \frac{d E(t)}{d t}=\frac{\beta S(t) I(t)}{\varphi(I)}-(\mu+\sigma) E(t) \\
& \frac{d I(t)}{d t}=\sigma E(t)-(\mu+\gamma) I(t) \\
& \frac{d V(t)}{d t}=\rho \pi-\gamma I(t)-(\mu+\omega) V(t)
\end{aligned}
$$

Where $\pi$ is the recruitment of individuals, that includes new born and immigrants into the susceptible population. $\rho$ is the fraction of the recruited individuals who are vaccinated, $\beta$ is the rate at which susceptible individuals becomes infected, $\mu$ is the natural death rate, $\sigma$ is the rate at which exposed individuals becomes infectious $\gamma$ is the rate at which infectious individuals are treated or recovered and $\omega$ is the rate at which vaccine wanes.

They considered a non-linear incidence rate of the form $\frac{\beta S(t) I(t)}{\varphi(I)}$. If $\varphi(I)=1$, then the incidence rate is bilinear. Let the function $\varphi(I)$ satisfy $\varphi(0)=1$ and $\varphi(0) \geq 0$, which implies that $\varphi(I) \geq 1$. then $\frac{1}{\varphi(I)}$ will be used to measure the psychological or inhibition effect of behavioural changes of the susceptible individuals when there is an increase in the number of infectious individuals. When the rate of infection is high, measures are put in place to reduce contact such as quarantine or protective measures by susceptible individuals thus the effective contacts between infected individuals and susceptible individuals decrease when the rate of infection is at high infective levels due to the quarantine of infected individuals or due to the protection measures by the susceptible individuals. [6]

To obtain the total population 
$N=(S(t)+E(t)+I(t)+V(t))$ Sum equations (1.1), (1.2), (1.3) and (1.4) to get.

$\frac{d N}{d t}=\pi-\rho \pi-\frac{\beta S I}{\varphi(t)}-\mu S+\omega V+\frac{\beta S I}{\varphi(t)}-(\mu+\sigma)+\sigma E-(\mu+\gamma) I+\rho \pi-\mu V+\gamma I-\omega V$

$\frac{d N}{d t}=\pi-\mu(S+E+I+V)$

$\frac{d N}{d t}=\pi-\mu N$

The initial population is set to be

$N_{o}=S_{o}+E_{o}+I_{o}+V_{o}=\frac{\pi}{\mu}$

In order to have a population of constant size, i.e.

$S(t)+E(t)+I(t)+V(t)=N \equiv \frac{\pi}{\mu}$

The stated variables $(S(t), E(t), I(t), V(t))$ remain biologically meaningful set.

$\Omega_{1}=\left\{(S, E, I, V) \in \mathrm{R}_{+}^{4} \backslash 0 \leq S+E+I+V \leq \frac{\pi}{\mu}\right\}$

For $(S(0), E(0), I(0), V(0)) \in \mathfrak{R}_{+}^{4}$, which is a positive variant region.

Using $V(t)=\frac{\pi}{\mu}-S(t)-E(t)-I(t)$ to eliminate $V(t)$

Equation (1.1), (1.2), (1.3) and (1.4) becomes

$\frac{d S}{d t}=(1-\rho) \pi-\frac{\beta S I}{\varphi(I)}-\mu S+\omega\left(\frac{\pi}{\mu}-S-E-I\right)$

$\frac{d E}{d t}=\frac{\beta S I}{\varphi(I)}-(\mu+\sigma) E$

$\frac{d I}{d t}=\sigma E-(\mu+\gamma) I$

Which reduces it to a three dimensional model. The total population $N_{1}$ becomes $N_{1}=S+E+I$

$\frac{d N_{1}}{d t}=\pi-\rho \pi-\frac{\beta S I}{\varphi(I)}-\mu S+\omega \frac{\pi}{\mu}-\omega S-\omega E-\omega I+\frac{\beta S I}{\varphi(I)}-\mu E-\sigma E+\sigma E-\mu I-\gamma I$

$\frac{d N_{1}}{d t}=\frac{\pi[(1-\rho)+\omega]}{\mu}-(\mu+\omega) N_{1}-\gamma I(t)$

From the above equation it can be seen that in the absence of the disease i.e. $I=0$ this implies $\gamma I=0$

$\frac{\pi[(1-\rho)+\omega]}{\mu}-(\mu+\omega) N_{1}=0$

$N_{1}=\frac{(\mu(1-\rho)+\omega) \pi}{\mu(\mu+\omega)}$ ast $\rightarrow \infty$

The spread of the disease in population will reduce $N_{1}$, this implies that.

$N_{1} \in\left(0, \frac{(\mu(1-\rho)+\omega) \pi}{\mu(\mu+\omega)}\right)$

Thus the new positively invariant region would be

$\Omega_{2}=\left((S, E, I) \in \mathfrak{R}^{3}: S \geq 0, I \geq 0, S+E+I \leq \frac{(\mu(1-\rho)+\omega) \pi}{\mu(\mu+\omega)}\right)$ 


\section{Existence And Stability Of Equilibria}

The existence and stability of equilibrium of models in equations (1.5), (1.6), and (1.7) are discussed, at equilibrium the model becomes

$$
\begin{aligned}
& (1-\rho) \pi-\frac{\beta S I}{\varphi(I)}-\mu S+\frac{\omega \pi}{\mu}-\omega S-\omega E-\omega I=0 \\
& \frac{\beta S I}{\varphi(I)}-(\mu+\sigma) E=0 \\
& \sigma E-(\mu+\gamma) I=0
\end{aligned}
$$

From these equations it can be seen that to have a diseases free equilibrium i.e. $I=0$ which implies $I=0$ From equation (1.9)

$$
\begin{aligned}
& (1-p) \pi-(\mu+\omega) S+\frac{\omega \pi}{\mu}=0 \\
& S=\frac{[(1-\rho)+\omega] \pi}{\mu(\mu+\omega)}
\end{aligned}
$$

From equation (1.10) $I=0$

$$
\begin{aligned}
& \frac{\beta S(0)}{\varphi(I)}-(\mu+\sigma) E=0 \\
& E=0
\end{aligned}
$$

The disease free equilibrium of equations (1.9), (1.10), and (1.11) is

$$
P_{o}=\left(\frac{[(1-\rho)+\omega] \pi}{\mu(\mu+\omega)}, 0,0\right)
$$

The other equilibrium points from (1.10), and (1.11) are as follows

From (1.10) we have

$$
S=\frac{(\mu+\sigma) E}{\beta I} \varphi(I)
$$

From equation (1.11) we have

$$
E=\frac{(\mu+\gamma) I}{\sigma}
$$

Substituting $E$ in $\mathrm{S}$ above to have

$$
S=\frac{(\mu+\sigma)(\mu+\gamma)}{\beta} \varphi(I)
$$

Substituting equations (1.12) and (1.13) into equation (1.9)

$$
H(I)=\frac{[\mu(1-\rho)+\omega] \pi}{\mu}-\left[\frac{(\mu+\sigma+\omega)(\mu+\gamma)+\sigma \omega}{\sigma}\right] I-\left[\frac{(\mu+\omega)(\mu+\sigma)(\mu+\gamma)}{\beta \sigma}\right] \varphi(I)
$$

It can be easily seen that the function $H(I)$ is negative for large positive $I$. Next, the sign of its derivatives were determined as:

$$
H(I)=-\left[\frac{(\mu+\sigma+\omega)(\mu+\gamma)+\sigma \omega}{\sigma}\right]-\left[\frac{(\mu+\omega)(\mu+\sigma)(\mu+\gamma)}{\beta \sigma}\right] \varphi(I)<0
$$

Since $\varphi(I) \geq 0$

$$
\mathfrak{R}_{o}=\frac{\beta \sigma(\mu(1-\rho)+\omega) \pi}{\mu(\mu+\omega)(\mu+\sigma)(\mu+\gamma)} \text { Since } \varphi(I)=1
$$

The compution of the Basic Reproduction Number of the disease free equilibrium is given in table 1 below. 
Table 1: Basic Reproductive Number of the Disease Free Equilibrium

\begin{tabular}{|l|c|c|c|c|c|c|c|l|}
\hline$\pi$ & $\beta$ & $\mu$ & $\gamma$ & $\omega$ & $\rho$ & $\sigma$ & $\Re_{o}$ & Remark \\
\hline 0.2 & 0.1 & 0.2 & 0.5 & 1.0 & 0.1 & 0.1 & 0.0468 & Globally Asymptotically Stable \\
\hline 0.2 & 0.2 & 0.2 & 0.5 & 0.9 & 0.1 & 0.2 & 0.1403 & Globally Asymptotically Stable \\
\hline 0.2 & 0.3 & 0.2 & 0.5 & 0.8 & 0.1 & 0.3 & 0.2520 & Globally Asymptotically Stable \\
\hline 0.2 & 0.4 & 0.2 & 0.5 & 0.7 & 0.1 & 0.4 & 0.3725 & Globally Asymptotically Stable \\
\hline 0.2 & 0.5 & 0.2 & 0.5 & 0.6 & 0.1 & 0.5 & 0.4974 & Globally Asymptotically Stable \\
\hline 0.2 & 0.6 & 0.2 & 0.5 & 0.5 & 0.1 & 0.6 & 0.6245 & Globally Asymptotically Stable \\
\hline 0.2 & 0.7 & 0.2 & 0.5 & 0.4 & 0.1 & 0.7 & 0.7519 & Globally Asymptotically Stable \\
\hline 0.2 & 0.8 & 0.2 & 0.5 & 0.3 & 0.1 & 0.8 & 0.8777 & Globally Asymptotically Stable \\
\hline 0.2 & 0.9 & 0.2 & 0.5 & 0.2 & 0.1 & 0.9 & 0.9944 & Globally Asymptotically Stable \\
\hline 0.2 & 1.0 & 0.2 & 0.5 & 0.1 & 0.1 & 1.0 & 1.1111 & Unstable \\
\hline
\end{tabular}

If we let $\varphi(I)=1$ then we'll have $I$ as

$$
\begin{aligned}
& I=\frac{1}{\sigma(\mu+\sigma)(\mu+\gamma)+\omega(\mu+\gamma)+\sigma \omega}\left[\pi\left\{(1-\rho)+\frac{\omega}{\mu}\right\}-(\mu+\omega)\left\{\frac{(\mu+\sigma)(\mu+\gamma)}{\beta \sigma}\right\}\right] \\
& E=\frac{(\mu+\gamma)}{\sigma} \frac{1}{\sigma(\mu+\sigma)(\mu+\gamma)+\omega(\mu+\gamma)+\sigma \omega}\left[\pi\left\{(1-\rho)+\frac{\omega}{\mu}\right\}-(\mu+\omega)\left\{\frac{(\mu+\sigma)(\mu+\gamma)}{\beta} \varphi(I)\right\}\right]
\end{aligned}
$$

\section{Stability Analysis Of The Equilibrium States}

Using the Jacobian determinant of the system of equations, we have

$$
J=\left|\begin{array}{cccc}
-\left(\frac{\beta I}{\varphi(I)}+\mu\right)-\lambda & 0 & -\frac{\beta S}{\varphi(I)} & \omega \\
\frac{\beta I}{\varphi(I)} & -(\mu+\sigma)-\lambda & \frac{\beta S}{\varphi(I)} & 0 \\
0 & \sigma & -(\mu+\gamma)-\lambda & 0 \\
0 & 0 & \gamma & -(\mu+\omega)-\lambda
\end{array}\right|
$$

At zero equilibrium state $(S, E, I, V)=(0,0,0,0)$. We have

$$
\begin{aligned}
& \therefore \lambda_{1}=-\mu \\
& \lambda_{2}=-(\mu+\sigma) \\
& \lambda_{3}=-(\mu+\gamma) \\
& \lambda_{4}=-(\mu+\omega) \\
& \lambda_{1}, \lambda_{2}, \lambda_{3} \text { and } \lambda_{4}<0
\end{aligned}
$$

The zero equilibrium state is stable.

Using the Jacobian determinant

$$
\begin{aligned}
J & =\left(\frac{\beta I}{\varphi(I)}+\mu\right)(\mu+\sigma)(\mu+\gamma)(\mu+\omega)+\left(\frac{\beta I}{\varphi(I)}+\mu\right)(\mu+\sigma)(\mu+\gamma) \lambda+\left(\frac{\beta I}{\varphi(I)}+\mu\right)(\mu+\sigma)(\mu+\omega) \lambda \\
& +\left(\frac{\beta I}{\varphi(I)}+\mu\right)(\mu+\sigma) \lambda^{2}+\left(\frac{\beta I}{\varphi(I)}+\mu\right)(\mu+\gamma)(\mu+\omega) \lambda+\left(\frac{\beta I}{\varphi(I)}+\mu\right)(\mu+\gamma) \lambda^{2}+\left(\frac{\beta I}{\varphi(I)}+\mu\right)(\mu+\omega) \lambda^{2} \\
& +\left(\frac{\beta I}{\varphi(I)}+\mu\right) \lambda^{3}+\left(\frac{\beta I}{\varphi(I)}+\mu\right)\left(\frac{\beta S}{\varphi(I)}\right)(\mu+\omega) \sigma+\left(\frac{\beta I}{\varphi(I)}+\mu\right)\left(\frac{\beta S}{\varphi(I)}\right) \sigma \lambda+(\mu+\sigma)(\mu+\gamma)(\mu+\omega) \lambda \\
& +(\mu+\sigma)(\mu+\gamma) \lambda^{2}+(\mu+\sigma)(\mu+\omega) \lambda^{2}+(\mu+\sigma) \lambda^{3}+(\mu+\gamma)(\mu+\omega) \lambda^{2}+(\mu+\gamma) \lambda^{3}+(\mu+\omega) \lambda^{3}+\lambda^{4} \\
& -\left(\frac{\beta S}{\varphi(I)}\right)(\mu+\omega) \sigma \lambda-\left(\frac{\beta S}{\varphi(I)}\right) \sigma \lambda^{2}+\left(\frac{\beta S}{\varphi(I)}\right)\left(\frac{\beta I}{\varphi(I)}\right)(\mu+\omega) \sigma+\left(\frac{\beta S}{\varphi(I)}\right)\left(\frac{\beta I}{\varphi(I)}\right) \sigma \lambda-\omega\left(\frac{\beta I}{\varphi(I)}\right) \sigma \gamma
\end{aligned}
$$




$$
\begin{aligned}
H(\lambda) & =\lambda^{4}+\left[\left(\frac{\beta I}{\varphi(I)}+\mu\right)+(\mu+\sigma)+(\mu+\gamma)+(\mu+\omega)\right] \lambda^{3} \\
& +\left[\left(\frac{\beta I}{\varphi(I)}+\mu\right)\{(\mu+\sigma)+(\mu+\gamma)+(\mu+\omega)\}+(\mu+\sigma)(\mu+\gamma)+(\mu+\sigma)(\mu+\omega)+(\mu+\gamma)(\mu+\omega)-\left(\frac{\beta S}{\varphi(I)}\right) \sigma\right] \lambda^{2} \\
& +\left[\left(\frac{\beta I}{\varphi(I)}+\mu\right)\left\{(\mu+\sigma)(\mu+\gamma)+(\mu+\sigma)(\mu+\omega)+(\mu+\gamma)(\mu+\omega)+\left(\frac{\beta S}{\varphi(I)}\right) \sigma\right\}+(\mu+\sigma)(\mu+\gamma)(\mu+\omega)\right] \lambda \\
& +\left[\left(\frac{\beta S}{\varphi(I)}\right) \sigma\left\{(\mu+\omega)+\left(\frac{\beta I}{\varphi(I)}\right)\right\}\right.
\end{aligned}
$$

Then applying the Bellman and Cooke's Theorem. We let $\lambda=i q$

$$
\begin{aligned}
& H(i q)=q^{4}+\left[\left(\frac{\beta I}{\varphi(I)}+\mu\right)+(\mu+\sigma)+(\mu+\gamma)+(\mu+\omega)\right] i q^{3} \\
& +\left[\left(\frac{\beta I}{\varphi(I)}+\mu\right)\{(\mu+\sigma)+(\mu+\gamma)+(\mu+\omega)\}+(\mu+\sigma)(\mu+\gamma)+(\mu+\sigma)(\mu+\omega)+(\mu+\gamma)(\mu+\omega)-\left(\frac{\beta S}{\varphi(I)}\right) \sigma\right] i q^{2} \\
& +\left[\left(\frac{\beta I}{\varphi(I)}+\mu\right)\left\{(\mu+\sigma)(\mu+\gamma)+(\mu+\sigma)(\mu+\omega)+(\mu+\gamma)(\mu+\omega)+\left(\frac{\beta S}{\varphi(I)}\right) \sigma\right\}\right] i q \\
& +\left[\begin{array}{l}
+(\mu+\sigma)(\mu+\gamma)(\mu+\omega)-\left(\frac{\beta S}{\varphi(I)}\right) \sigma\left\{(\mu+\omega)+\left(\frac{\beta I}{\varphi(I)}\right)\right\} \\
+
\end{array}\right. \\
& +\left[\left(\frac{\beta I}{\varphi(I)}+\mu\right)(\mu+\omega)\left\{(\mu+\sigma)(\mu+\gamma)+\left(\frac{\beta S}{\varphi(I)}\right) \sigma\right\}+\left(\frac{\beta I}{\varphi(I)}\right) \sigma\left\{\left(\frac{\beta S}{\varphi(I)}\right)(\mu+\omega)-\omega \gamma\right\}\right]
\end{aligned}
$$

Resolving equation (1.16) into real and imaginary parts, we'll obtain

$$
\begin{aligned}
& H(q)=F(q)+i G(q) \\
& F(q)=q^{4}+\left[\begin{array}{l}
\left(\frac{\beta I}{\varphi(I)}+\mu\right)\{(\mu+\sigma)+(\mu+\gamma)+(\mu+\omega)\}+(\mu+\sigma)(\mu+\gamma)+(\mu+\sigma)(\mu+\omega) \\
+(\mu+\gamma)(\mu+\omega)-\left(\frac{\beta S}{\varphi(I)}\right) \sigma
\end{array}\right] q^{2} \\
& +\left[\left(\frac{\beta I}{\varphi(I)}+\mu\right)(\mu+\omega)\left\{(\mu+\sigma)(\mu+\gamma)+\left(\frac{\beta S}{\varphi(I)}\right) \sigma\right\}+\left(\frac{\beta I}{\varphi(I)}\right) \sigma\left\{\left(\frac{\beta S}{\varphi(I)}\right)(\mu+\omega)-\omega \gamma\right\}\right] \\
& G(q)=i q^{3}\left[\left(\frac{\beta I}{\varphi(I)}+\mu\right)+(\mu+\sigma)+(\mu+\gamma)+(\mu+\omega)\right]+ \\
& +\left[\begin{array}{l}
\left.\left(\frac{\beta I}{\varphi(I)}+\mu\right)\left\{(\mu+\sigma)(\mu+\gamma)+(\mu+\sigma)(\mu+\omega)+(\mu+\gamma)(\mu+\omega)+\left(\frac{\beta S}{\varphi(I)}\right) \sigma\right\}\right] \\
+(\mu+\sigma)(\mu+\gamma)(\mu+\omega)-\left(\frac{\beta S}{\varphi(I)}\right) \sigma\left\{(\mu+\omega)+\left(\frac{\beta I}{\varphi(I)}\right)\right\}
\end{array}\right] i q \\
& F^{\prime}(q)=4 q^{3}+2 q\left[\begin{array}{l}
\left(\frac{\beta I}{\varphi(I)}+\mu\right)\{(\mu+\sigma)+(\mu+\gamma)+(\mu+\omega)\}+(\mu+\sigma)(\mu+\gamma) \\
+(\mu+\sigma)(\mu+\omega)+(\mu+\gamma)(\mu+\omega)-\left(\frac{\beta S}{\varphi(I)}\right) \sigma
\end{array}\right]
\end{aligned}
$$




$$
\begin{aligned}
G^{\prime}(q)= & 3 q^{2}\left[\left(\frac{\beta I}{\varphi(I)}+\mu\right)+(\mu+\sigma)+(\mu+\gamma)+(\mu+\omega)\right]+ \\
& +\left[\begin{array}{l}
\left.\left(\frac{\beta I}{\varphi(I)}+\mu\right)\left\{(\mu+\sigma)(\mu+\gamma)+(\mu+\sigma)(\mu+\omega)+(\mu+\gamma)(\mu+\omega)+\left(\frac{\beta S}{\varphi(I)}\right) \sigma\right\}\right] \\
+(\mu+\sigma)(\mu+\gamma)(\mu+\omega)-\left(\frac{\beta S}{\varphi(I)}\right) \sigma\left\{(\mu+\omega)+\left(\frac{\beta I}{\varphi(I)}\right)\right\}
\end{array}\right.
\end{aligned}
$$

When $q=0$

$$
\begin{aligned}
F(0)= & \left(\frac{\beta I}{\varphi(I)}+\mu\right)(\mu+\omega)\left\{(\mu+\sigma)(\mu+\gamma)+\left(\frac{\beta S}{\varphi(I)}\right) \sigma\right\}+\left(\frac{\beta I}{\varphi(I)}\right) \sigma\left\{\left(\frac{\beta S}{\varphi(I)}\right)(\mu+\omega)-\omega \gamma\right\} \quad(0.19) \\
G(0)= & 0 \\
F^{\prime}(0)= & 0 \\
G^{\prime}(0)= & \left(\frac{\beta I}{\varphi(I)}+\mu\right)\left\{(\mu+\sigma)(\mu+\gamma)+(\mu+\sigma)(\mu+\omega)+(\mu+\gamma)(\mu+\omega)+\left(\frac{\beta S}{\varphi(I)}\right) \sigma\right\}+(\mu+\sigma)(\mu+\gamma)(\mu+\omega) \\
& -\left(\frac{\beta S}{\varphi(I)}\right) \sigma\left\{(\mu+\omega)+\left(\frac{\beta I}{\varphi(I)}\right)\right\}
\end{aligned}
$$

Substituting $\mathrm{F}(0)$ and $\mathrm{G}^{\prime}(0)$ into the conditions Stated in the Bellman and Cooke's Theorem $J=F(0) G^{\prime}(0)>0$

Hence the condition holds for $J=0$

Substitute equation $(1.13,1.15$ and 1.16$)$ into $\mathrm{F}(0)$ and $\mathrm{G}^{\prime}(0)$ and then computed $J$ with MATLAB. We have the table below.

Table 2: The Result of the Stability Analysis of the Epidemic Free Equilibrium

Table 2: The Result of the Stability Analysis of the Epidemic Free Equilibrium
\begin{tabular}{|l|c|c|c|c|c|c|c|l|}
\hline$\pi$ & $\beta$ & $\mu$ & $\gamma$ & $\omega$ & $\rho$ & $\sigma$ & $J$ & Remark \\
\hline 0.2 & 0.1 & 0.2 & 0.5 & 1.0 & 0.1 & 0.1 & 2.1763 & Stable \\
\hline 0.2 & 0.2 & 0.2 & 0.5 & 0.9 & 0.1 & 0.2 & 0.6623 & Stable \\
\hline 0.2 & 0.3 & 0.2 & 0.5 & 0.8 & 0.1 & 0.3 & 0.2825 & Stable \\
\hline 0.2 & 0.4 & 0.2 & 0.5 & 0.7 & 0.1 & 0.4 & 0.1230 & Stable \\
\hline 0.2 & 0.5 & 0.2 & 0.5 & 0.6 & 0.1 & 0.5 & 0.0470 & Stable \\
\hline 0.2 & 0.6 & 0.2 & 0.5 & 0.5 & 0.1 & 0.6 & 0.0124 & Stable \\
\hline 0.2 & 0.7 & 0.2 & 0.5 & 0.4 & 0.1 & 0.7 & 0.0089 & Stable \\
\hline 0.2 & 0.8 & 0.2 & 0.5 & 0.3 & 0.1 & 0.8 & 0.0021 & Stable \\
\hline 0.2 & 0.9 & 0.2 & 0.5 & 0.2 & 0.1 & 0.9 & $-0.24225 \mathrm{e}^{-4}$ & Unstable \\
\hline 0.2 & 1.0 & 0.2 & 0.5 & 0.1 & 0.1 & 1.0 & $-1.3261 \mathrm{e}^{-5}$ & Unstable \\
\hline
\end{tabular}

\section{Conclusion}

This paper is on a vaccination model with nonlinear incidence rate and vaccination waning period, the basic reproduction number $\mathfrak{R}_{o}$ of the disease free equilibrium was analyzed, the result shows that the disease free equilibrium is globally asymptotically stable, when the recruitment of individuals into the susceptible class $\pi$ is a constant 0.2 , the natural death rate $\mu$ is a constant 0.2 , the contact rate at which susceptible becomes infected $\beta$ varies from 0.1 to 1.0 , the rate at which infected individuals recover or are treated is a constant 0.5 , the fraction of recruited that are vaccinated $\rho$ is a constant 0.1 , the rate at which exposed individuals becomes infectious varies from $0.1-1.0$ and the vaccination waning period varies from $1.0-0.2$ and unstable at the point 0.1 . This conclusion was reached based on a theorem which states that: If $\Re_{o}<1$ then the disease-free equilibrium $P_{o}$ is globally asymptotically stable, if $\mathfrak{R}_{o}=1, P_{o}$ is stable, if $\mathfrak{R}_{o}>1, P_{o}$ is unstable.

The epidemic free equilibrium was also analyzed using the same conditions as the disease free equilibrium, it was found to be stable, on the probability points of the disease waning periods from 1.0 down to 
0.3 and unstable on points 0.2 and 0.1 . Which is logical, that is, when the vaccine efficacy reduces we expect that the stability of the model should reduce. The need for this paper is based on the fact that the work of L-M. Cai and X- Z. Li. [6] was not simulated which make it hard to see the application interpretation of the model, but when simulated numerically we are able to see how variations in rate of the variables affect the outcome of the results. From the outcome of this paper, it is therefore recommended that people should be vaccinated before the disease wane out and that more research should be done on how to increases the disease waning period, so as to prolong the effectiveness of the vaccines.

\section{References}

[1] R. Naresh, S. Panduy, A.K. Misra, Analysis of Vaccination model for carrier dependent infectious diseases with environmental effects. Journal of Non-linear Analysis: Modeling and control, 13 (3), 2008, 331-350.

[2] C.P. Farrington, On Vaccine Efficacy and Reproduction numbers, Journal of Mathematics and Bioscience, 185, 2003 89-109.

[3] A.B. Gumel, S.M. Moghadas, A qualitative Study of a Vaccination Model with Non-Linear Incidence. Journal of Appl. Math. Comp, 143, 2003, 409-419.

[4] C.M. Kribs-Zaleta, J.X. Velasco-Hernandez, A Simple Vaccination Model With Multiple Epidemic States, Math. Biosc. 164, 2000, 183-201.

[5] B. shulgin, L. Stone, Z. Agur, Pulse Vaccination Strategy in the SIR Epidemic Model. Bull Math. Biol. (1998) 60, pp. 1123-1148.

[6] L-M. Cai, X-Z. Li, Analysis of a SEIV Epidemic Model With Non-Linear Incidence Rate, Applied Mathematical Modelling (2008), doi: 10.1016/j.apm.2008.01.005

[7] J.K. Matt and R. Pejman, Modelling Infectious Diseases in Human and Animals, New Jersey, Princeton and Oxford, 2008.

[8] J. Jia and ping Li, Global Analysis of Human SVEIR Epidemic Model with Partial Immunity, Mathematica Aeterna, (2011), Vol 1 pp. 547-561

[9] M. De La Sen, S. Alonso-Quesada and A. Ibeas, Observer based Vaccination for a SEIR Epidemic Model with Time Varying Total Population, International Conference on Circuits, Systems and Simulation (IPCSIT), (2011) Vol. 7, pp.11-22. IACSIT Press Singapore.

[10] M. De La Sen, S. Alonso-Quesada and A. Ibeas, On the Equilibrium Points and Some Properties of a SVEIRS Epidemic Model. Proceedings of the World Congress on Engineering (2011) Vol. III, London UK.

[11] J. Arino, C.C. Mccluskey and P. Van Den Driessche, Global Result for an Epidemic Model with Vaccination that Exhibits Backward Bifurcation. SIAM Journal of Applied Mathematics. 64 (1), 2004, 260-276

[12] S. Singh, P. Chandra, J.B. Shukla, Modelling and analysis of the spread of carrier dependent infectious diseases with environmental effects, J. Boil. Sys., 11(3), 2003, 325-335

[13] M. Ghosh, P. Chandra, P. Sinha, J.B. Shukla, Modelling the Spread of Carrier - Dependent infectious Diseases with environmental Effects, Appl. Math. Comp., 152 2004, 385 - 402 